\title{
High sensitivity pyrogen testing in water and dialysis solutions
}

\author{
Mardas Daneshian ${ }^{\mathrm{a}}$, Albrecht Wendel ${ }^{\mathrm{a}}$, Thomas Hartung ${ }^{\mathrm{a}, \mathrm{b}}$, Sonja von Aulock ${ }^{\mathrm{a}, *}$ \\ aiochemical Pharmacology, University of Konstanz, Germany \\ ECVAM, JRC, Ispra, Italy
}

\section{A R T I C L E I N F O}

\section{Article history:}

Received 4 June 2007

Received in revised form 25 March 2008

Accepted 28 March 2008

Available online 24 April 2008

\section{Keywords:}

Dialysis fluids

Interleukin-1[beta]

Lipopolysaccharide

Pyrogen testing

Whole blood test

\begin{abstract}
A B S T R A C T
Background: The dialysis patient is confronted with hundreds of litres of dialysis solution per week, which pass the natural protective barriers of the body and are brought into contact with the tissue directly in the case of peritoneal dialysis or indirectly in the case of renal dialysis (hemodialysis). The components can be tested for living specimens or dead pyrogenic (feverinducing) contaminations. The former is usually detected by cultivation and the latter by the endotoxin-specific Limulus Amoebocyte Lysate Assay (LAL). However, the LAL assay does not reflect the response of the human immune system to the wide variety of possible pyrogenic contaminations in dialysis fluids. Furthermore, the test is limited in its sensitivity to detect extremely low concentrations of pyrogens, which in their sum result in chronic pathologies in dialysis patients. The In vitro Pyrogen Test (IPT) employs human whole blood to detect the spectrum of pyrogens to which humans respond by measuring the release of the endogenous fever mediator interleukin-1 3 . Spike recovery checks exclude interference. The test has been validated in an international study for pyrogen detection in injectable solutions. Methods: In this study we adapted the IPT to the testing of dialysis solutions. Results: Preincubation of $50 \mathrm{ml}$ spiked samples with albumin-coated microspheres enhanced the sensitivity of the assay to detect contaminations down to $0.1 \mathrm{pg} / \mathrm{ml}$ LPS or $0.001 \mathrm{EU} / \mathrm{ml}$ in water or saline and allowed pyrogen detection in dialysis concentrates or final working solutions. Conclusions: This method offers high sensitivity detection of human-relevant pyrogens in dialysis solutions and components.
\end{abstract}

\section{Introduction}

The word "pyrogen" comes from the Greek word "pyros" meaning "fire". Pyrogens induce an inflammatory response characterized among other symptoms by a fever reaction. Most known pyrogens are of microbial origin. They include bacteria, yeasts and fungi and their components. The best known pathogen-associated molecular patterns (PAMPs) responsible for the pyrogenic reaction are lipopolysaccharide (LPS, endotoxin) from Gram-negative bacteria, lipoteichoic acid (LTA) and peptidoglycan (PGN) from Gram-positive bacteria, but also dusts and other organic and inorganic particles may have pyrogenic qualities.

* Corresponding author. Biochemical Pharmacology, University of Konstanz, P.O. Box M668, 78457 Konstanz, Germany. Tel.: +49 7531 882121; fax: +49 7531 884156.

E-mail address: Sonja.v-Aulock@uni-konstanz.de (S. von Aulock)
Renal dialysis patients are exposed to hundreds of litres of dialysis fluids every week with the dialysis membrane being the only barrier between the fluid and the blood stream. Although these membranes prevent whole bacteria from passing into the blood stream, they display variable efficiency in holding back pyrogens (Ledebo, 2004). The maximum pyrogen content of dialysis fluids is defined by various pharmacopoeias and the AAMI (Association for the Advancement of Medical Instrumentation) and ranges between 0.03 (ultrapure) and 2 endotoxin units per $\mathrm{ml}$ (Ward, 2004). These limits were likely defined based on the sensitivity of the detection methods available at the time. The use of ultrapure water is today considered state of the art (Ledebo, 2004).

The content of the best known and highly potent pyrogen LPS can be measured by Limulus Amoebocyte Lysate Assay (LAL). This method exploits the immune defence reaction of the Limulus crab's hemolymph, which coagulates when in 
contact with LPS. The most sensitive variants of this method detect $3 \mathrm{pg} / \mathrm{ml}$ LPS, i.e. $0.03 \mathrm{EU} / \mathrm{ml}$. However, this method has a number of drawbacks: other pyrogenic molecules are not detected; numerous substances interfere with the assay and the test reflects rather the amount of LPS than its actual inflammatory potential in humans. Regarding the latter point, we and others have shown that LPS from different species differ greatly in their immunostimulatory potential in humans (Fennrich et al., 1999; Darveau et al., 2004; Dehus et al., 2006).

The In vitro Pyrogen Test (IPT) is an alternative test which models the human fever reaction (Hartung and Wendel, 1996). In the human body, pyrogens are recognized by pathogen recognition receptors (PRR) on monocytes in the blood and macrophages in the tissues. In response they release proinflammatory cytokines such as IL-1 $\beta$, TNF $\alpha$ and IL- 6 into the blood stream. These bind to receptors on the blood-brain barrier and cause the release of prostaglandin $E_{2}$ in the brain, which in turn changes the temperature set-point in the hypothalamus, setting off the fever response (Dinarello, 2004). The IPT detects the release of the proinflammatory cytokine IL-1 $\beta$ in human whole blood in response to pyrogenic contaminations in test samples.

The test was originally developed for safety testing of parenterals and has been internationally validated for this application (Hoffmann et al., 2005; Schindler et al., 2006). Further developments include safety testing of biomaterials (Mazzotti et al., 2007), the evaluation of the inflammatory burden in the air (Kindinger et al., 2005), which is associated with the development of chronic obstructive lung diseases, and special adaptations for pyrogen testing in immunomodulatory or toxic medications, e.g. injectable cortisone or chemotherapeutics (Daneshian et al., 2006). In the latter study we described the use of albumin-linked beads to bind LPS or other pyrogens in the sample. This allowed the separation of the pyrogens from the interfering sample. The method was termed AWIPT (adsorption, washing and in-vitro pyrogen test).

In the present study we adapted the AWIPT further to allow the detection of extremely low concentrations of pyrogens in dialysis fluids by accumulating pyrogenic spikes added to large volumes of fluid on the albumin-linked beads. Furthermore, this adaptation allowed the detection of pyrogens in the dialysis concentrates and the workings solutions. This adaptation, termed AcWIPT (accumulation, washing and in vitro pyrogen test) shall allow a better evaluation of the total pyrogenic activity the patient is exposed to per dialysis cycle.

\section{Methods}

\subsection{Pyrogens}

Endotoxin (LPS) from Escherichia coli 0-113 (EC-6, a kind gift from Dr. S. Poole, National Institute of Biological Standard and Controls, Herts, UK) was used. $100 \mathrm{pg}$ of this LPS are defined as 1 endotoxin unit (EU) (Poole and Mussett, 1989). LTA from Staphylococcus aureus was isolated and prepared inhouse as described previously (Morath et al., 2001). Spike concentrations described in the text and figures always refer to the concentration in the sample solution. E. coli and $S$. aureus were cultivated in Luria-Bertani medium and harvested at the exponential growth stage.
Table 1

Composition of hemodialysis fluids

\begin{tabular}{lcccccc}
\hline Conc. [mM] & \multicolumn{5}{l}{ Hemodialysis fluids } & \\
\cline { 2 - 7 } & No. 204 & No. 278 & No. 294 & No. 751 & No. 787 & No. 200 \\
\hline $\mathrm{K}^{+}$ & 70 & 0 & 70 & 70 & 140 & 0 \\
$\mathrm{Ca}^{2+}$ & 61.25 & 43.75 & 61.25 & 52.5 & 52.5 & 0 \\
$\mathrm{Glucose}^{2}$ & 0 & 194.22 & 194.22 & 388.43 & 194.22 & 0 \\
$\mathrm{Cl}^{-}$ & 3885 & 3885 & 3885 & 3885 & 3885 & 0 \\
$\mathrm{Mg}^{2+}$ & 17.5 & 17.5 & 17.5 & 17.5 & 17.5 & 0 \\
$\mathrm{Na}^{+}$ & 3605 & 3605 & 3605 & 3605 & 3605 & 0 \\
$\mathrm{CO}_{3}^{2-}$ & 0 & 0 & 0 & 0 & 0 & 1000 \\
\hline
\end{tabular}

\subsection{Samples}

Hemodialysis fluids 204, 278, 294, 751, 787 and 200 as well as peritoneal dialysis fluids Gambrosol trio 10 and Gambrosol trio 40 were gifts from Gambro Healthcare, Stockholm, Sweden. Their contents are listed in Tables 1 and 2 . In the case of the hemodialysis fluids the working dilution is $1 / 35$. The glucose concentrations of peritoneal dialysis solutions were adjusted to $1.5 \%$ or $2.5 \%$ or $3.9 \%(\mathrm{w} / \mathrm{v})$, as administered to dialysis patients. The fluids and solutions were spiked in their working concentrations.

\subsection{In vitro pyrogen test (IPT)}

Heparinized blood was drawn from healthy donors by venous puncture. Differential blood cell counts were routinely performed to rule out acute infections (Pentra 60, ABX Diagnostics). $100 \mu \mathrm{l}$ test sample was diluted with $1 \mathrm{ml} 0.9 \%$ saline and incubated with $100 \mu \mathrm{l}$ blood over night at $37^{\circ} \mathrm{C}$ and $5 \% \mathrm{CO}_{2}$ in a $1.5 \mathrm{ml}$ polypropylene reaction vial. After incubation, samples were resuspended and spun down ( 2 min at $13,000 \mathrm{~g}$ ) and supernatants were stored at $-80{ }^{\circ} \mathrm{C}$ until cytokine measurement. Interleukin- $1 \beta$ was quantified using an in-house sandwich ELISA based on a matched antibody pair (R\&D Systems). Binding of biotinylated antibody was quantified using streptavidin-peroxidase (Biosource) and the substrate 3,3',5,5'-tetramethylbenzidine (Sigma-Aldrich). Recombinant IL-1 $\beta$ was a generous gift from Dr. S. Poole (NIBSC).

\subsection{Preparation of albumin beads}

Macroporous acrylic beads (Eupergit, Degussa) decorated with human serum albumin, fraction $\mathrm{V}$ (low endotoxin) (Serva, Heidelberg, Germany) via covalent carboxyl linkage as

Table 2

Composition of reconstituted peritoneal dialysis solutions

\begin{tabular}{lll}
\hline Conc. [mM] & \multicolumn{2}{l}{ Peritoneal dialysis solutions } \\
\cline { 2 - 3 } & Gambrosol trio 10 & Cambrosol trio 40 \\
\hline $\mathrm{Ca}^{2+}$ & 1.70 & 1.31 \\
$\mathrm{Cl}^{-}$ & 96.0 & 95.2 \\
$\mathrm{Mg}^{2+}$ & 0.24 & 0.24 \\
$\mathrm{Na}^{+}$ & 131.0 & 131.0 \\
Lactate & 39.0 & 39.0 \\
Glucose & 85 or 139 or 215 & 85 or 139 or 215 \\
\hline
\end{tabular}


described previously (Zimmermann et al., 1999; Zimmermann, 2000) were provided by Fresenius Hemocare (St. Wendel, Germany) in vacuum sealed, sterile cartridges. Approximately $2.5 \mathrm{~g}$ were transferred into $50 \mathrm{ml}$ polypropylene tubes (Greiner) and rinsed with $50 \mathrm{ml} 0.9 \%$ saline (Berlin Chemie) to remove residual unbound albumin. After sedimentation of the beads, the supernatant was removed. In initial experiments, the beads were rinsed with $50 \mathrm{ml}$ priming solution $\left(4 \mathrm{mM} \mathrm{K}{ }^{+}, 1.75 \mathrm{mM} \mathrm{Ca}^{2+}, 0.5 \mathrm{mM} \mathrm{Mg}^{2+}\right.$, $106.5 \mathrm{mM} \mathrm{Cl}^{-}, 134 \mathrm{mM} \mathrm{Na}^{+}, 36 \mathrm{mM} \mathrm{HCO}-5.7 \mathrm{mM}$ citrate, $6.2 \mathrm{mM}$ dextrose, $\mathrm{pH} 7.2$, provided by Fresenius Hemocare). $40 \mathrm{ml}$ of supernatant was removed and $2 \% \mathrm{v} / \mathrm{v}$ DMSO (Cryosure, WAK Chemie) was added. In later experiments, these steps were omitted and beads were used directly after sedimentation without further treatment.

\subsubsection{AWIPT (adsorption, washing and in-vitro pyrogen test)}

The AWIPT was performed as described (Daneshian et al., 2006).

Adsorption: $100 \mu \mathrm{l}$ bead suspension was incubated with $100 \mu \mathrm{l}$ sample or LPS spike in a $2 \mathrm{ml}$ reaction vial at $4{ }^{\circ} \mathrm{C}$ horizontally overnight.

Washing: $1 \mathrm{ml}$ saline (Sigma, cell culture grade) was added to the beads, then removed using a suction pump after sedimentation. This procedure was repeated three times with clinical saline solution.

IPT: the whole blood incubation protocol (see above) was adapted as follows: to each bead-containing tube, $250 \mu \mathrm{l}$ saline (Berlin Chemie) and $100 \mu \mathrm{l}$ heparinized blood of a healthy donor were added, and incubated over night at $37^{\circ} \mathrm{C}$ in the presence of $5 \% \mathrm{CO}_{2}$.

\subsubsection{AcWIPT (accumulation, washing and in vitro pyrogen test)}

Accumulation: $100 \mu \mathrm{l}$ bead suspension was transferred to a $50 \mathrm{ml}$ reaction tube (Greiner bio-one). $50 \mathrm{ml}$ sample with or without LPS spike was added to the beads, and the tube was rolled over night at room temperature to allow efficient adsorption. The washing and IPT procedure was identical to that described for the AWIPT.

\subsubsection{LAL (limulus amebocyte lysate test)}

LAL testing was performed by using a kinetic, chromogenic endpoint LAL kit (Charles River Endosafe, Charlston, SC, USA) following the manufacturer's instructions.

\subsection{Data analysis}

According to the standard operating procedure of the IPT, the IL-1 $\beta$ secretion in response to the LPS spike must be greater than the mean of the control samples plus three times their standard deviation for the test to be valid. A sample is deemed interfering with pyrogen detection if the sample spiked with LPS induces IL- $1 \beta$ release that is less than $50 \%$ or more than $200 \%$ of IL- $1 \beta$ released in response to the same spike concentration in the absence of sample.

\subsection{Statistics}

Data are presented as means \pm SEM. Statistical significance was determined using GraphPad Prism 3.0 (GraphPad software, San Diego, U.S.A.). Groups were compared by one-way
ANOVA followed by Dunnett's multiple comparison test. *, $p<0.05$; **, $p<0.01$ in comparison to the control.

\section{Results}

\subsection{Lack of requirement for priming of beads}

The original protocol developed for preparing the albumin beads required them to be "primed" before use by incubation and washing in a special solution. As this procedure is timeintensive and beads are lost by repeated washing, we evaluated whether this procedure is necessary to enable the beads to absorb pyrogens. Comparison of cytokine induction in whole blood induced by incubating previously unprimed or primed beads with different concentrations of LPS, LTA or zymosan and then with whole blood showed higher absolute amounts of IL- $1 \beta$ induced in the unprimed beads while the curve shapes were the same and the controls remained nonstimulatory (Fig. 1). Therefore, all further experiments were performed with unprimed beads.

\subsection{Highly sensitive detection of pyrogens by AcWIPT}

To test the sensitivity of the AcWIPT method in comparison to the AWIPT and the standard IPT, we incubated beads
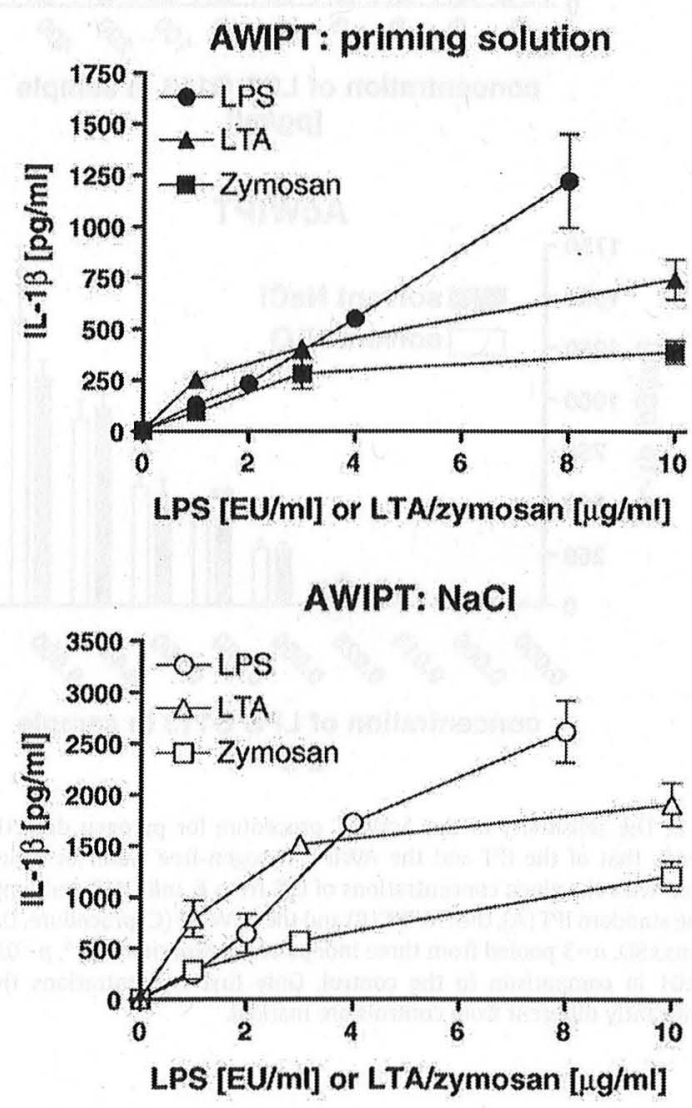

Fig. 1. Treatment of albumin-linked beads with priming solution does not increase LPS binding capacity. LPS from $E$. coli 0113 (in EU/ml, $1 \mathrm{EU}=100 \mathrm{pg}$ / $\mathrm{ml}$ ), LTA from $S$. aureus and Zymosan $\mathrm{A}$ (both in $\mu \mathrm{g} / \mathrm{ml}$ ) were detected by AWIPT after albumin-linked beads had (A) or had not (B) been treated with priming solution. Note the different scales of the $Y$-axis. Data are mean \pm range, $n=3$ representing three independent experiments. 

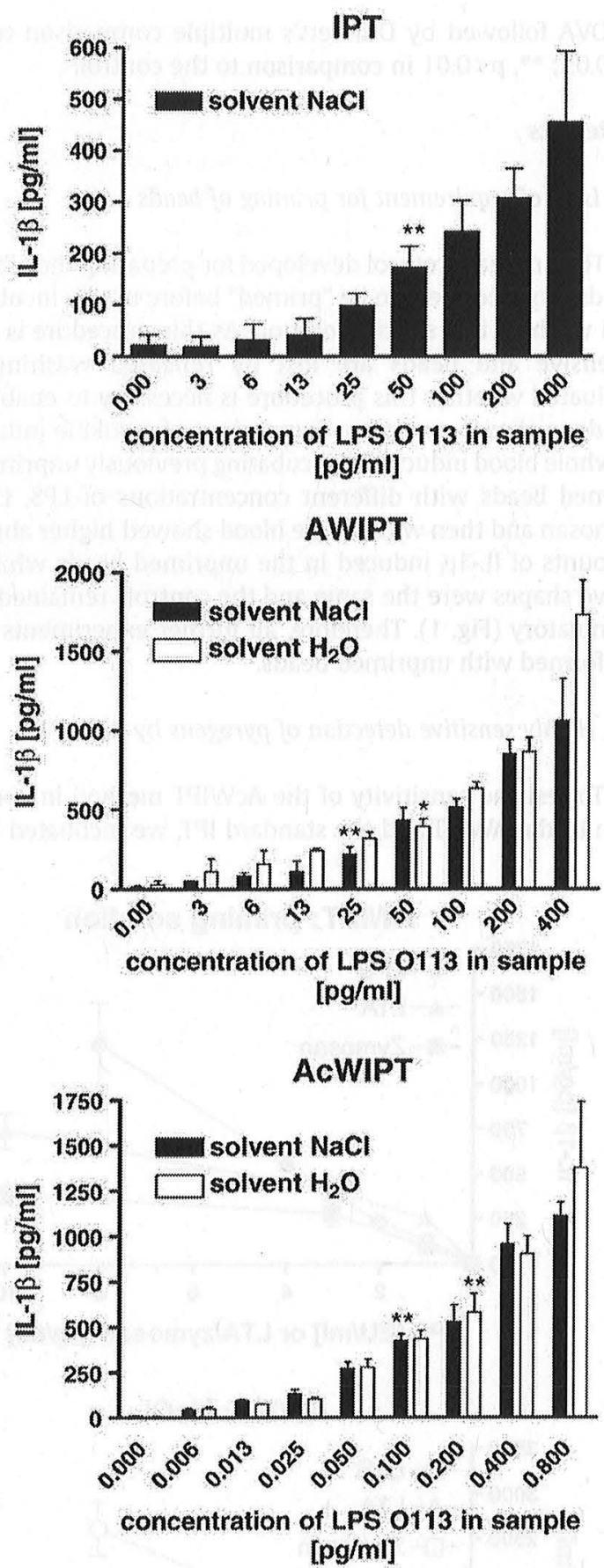

Fig. 2. The sensitivity of the AcWIPT procedure for pyrogen detection far exceeds that of the IPT and the AWIPT. Pyrogen-free water or saline was spiked with the given concentrations of LPS from E. coli 0113 and employed in the standard IPT (A), the AWIPT (B) and the AcWIPT (C) procedure. Data are means $\pm S D, n=3$ pooled from three independent experiments. *, $p<0.05$; **, $p<0.01$ in comparison to the control. Only first concentrations that are significantly different from controls are marked.

with a serial dilution series of LPS in water or saline according to the different developed protocols and measured IL-1 $\beta$ release in human blood. Although the AWIPT procedure led to increased levels of IL- $1 \beta$ release in comparison to the IPT, the sensitivity remained approximately the same $(25$ to $50 \mathrm{pg} / \mathrm{ml}$
LPS, i.e. 0.25 to $0.5 \mathrm{EU} / \mathrm{ml} \mathrm{LPS}$ ). Increasing the volume of sample combined with rolling during the preincubation step at room temperature allowed the detection of LPS spikes as low as 0.1 to $0.2 \mathrm{pg} / \mathrm{ml} \mathrm{LPS}$ or 0.001 or $0.002 \mathrm{EU} / \mathrm{ml}$ in saline or water respectively (Fig. 2).

3.3. Spike recovery of LPS in diluted dialysis concentrates and solutions by AcWIPT

Hemodialysis concentrates interfere with pyrogen testing, probably due to their osmolarity and potassium content known to inhibit caspase-1, which processes pro-IL-1 $\beta$ to IL$1 \beta$ for secretion (Walev et al., 2000). The AcWIPT procedure enabled LPS spike recovery in two of the undiluted hemodialysis concentrates tested and of the LTA spike in all
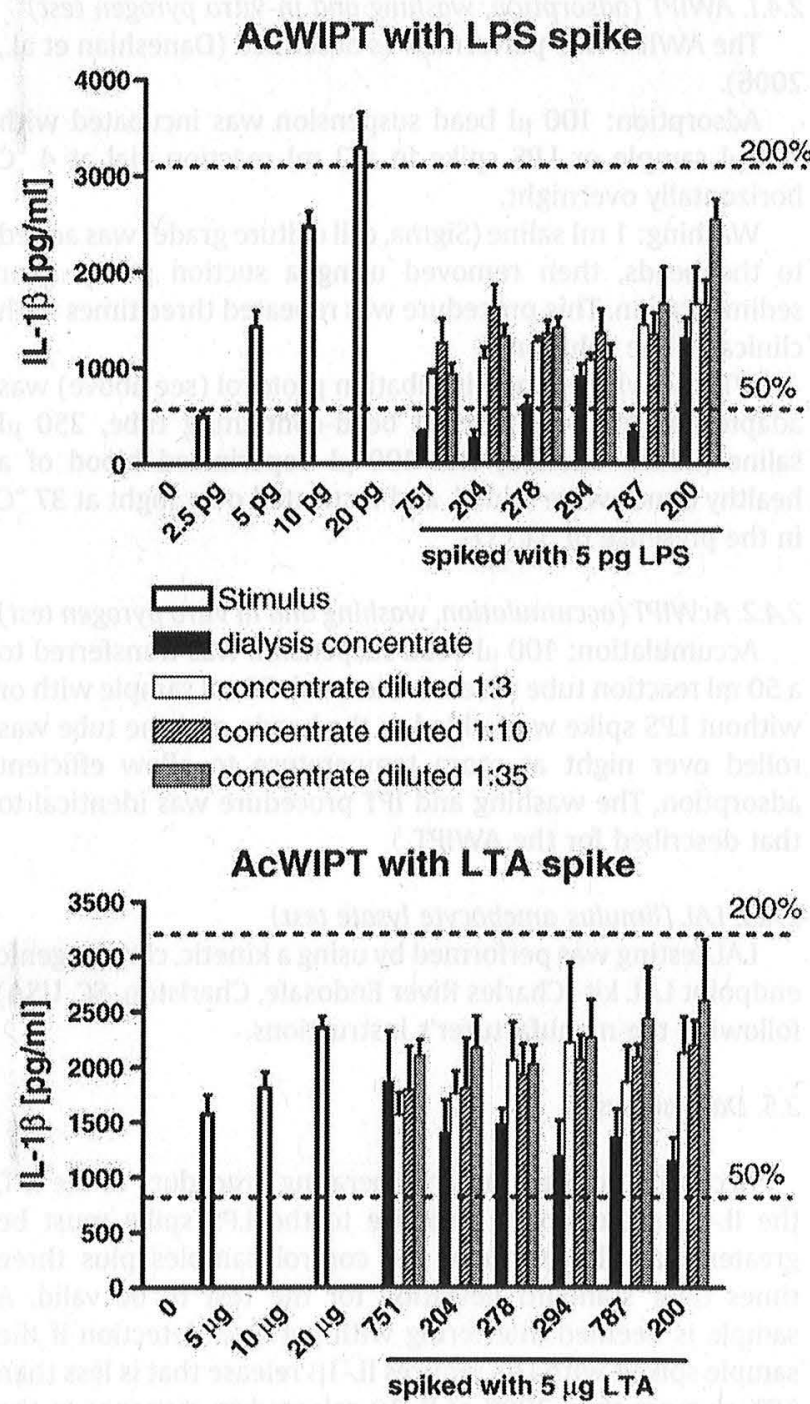

Fig. 3. Sensitive spike retrieval in some hemodialysis concentrates and all tested dialysis working solutions by AcWIPT. $50 \mathrm{ml}$ dialysis concentrates and $1 / 3,1 / 10$ and $1 / 35$ dilution spiked with 5 pg LPS (A), or $5 \mu \mathrm{g}$ LTA (B) were employed in the AcWIPT. In parallel, an LPS or LTA concentration response curve was performed in the AcWIPT. The dotted line shows $50 \%$ and $200 \%$ spike retrieval. Data are mean \pm range, $n=3$ from three independent experiments. IL-1 $\beta$ induction by all unspiked samples was below the detection limit of the ELISA $(3.4 \mathrm{pg} / \mathrm{ml})$. 


\section{AcWIPT with LPS spike}
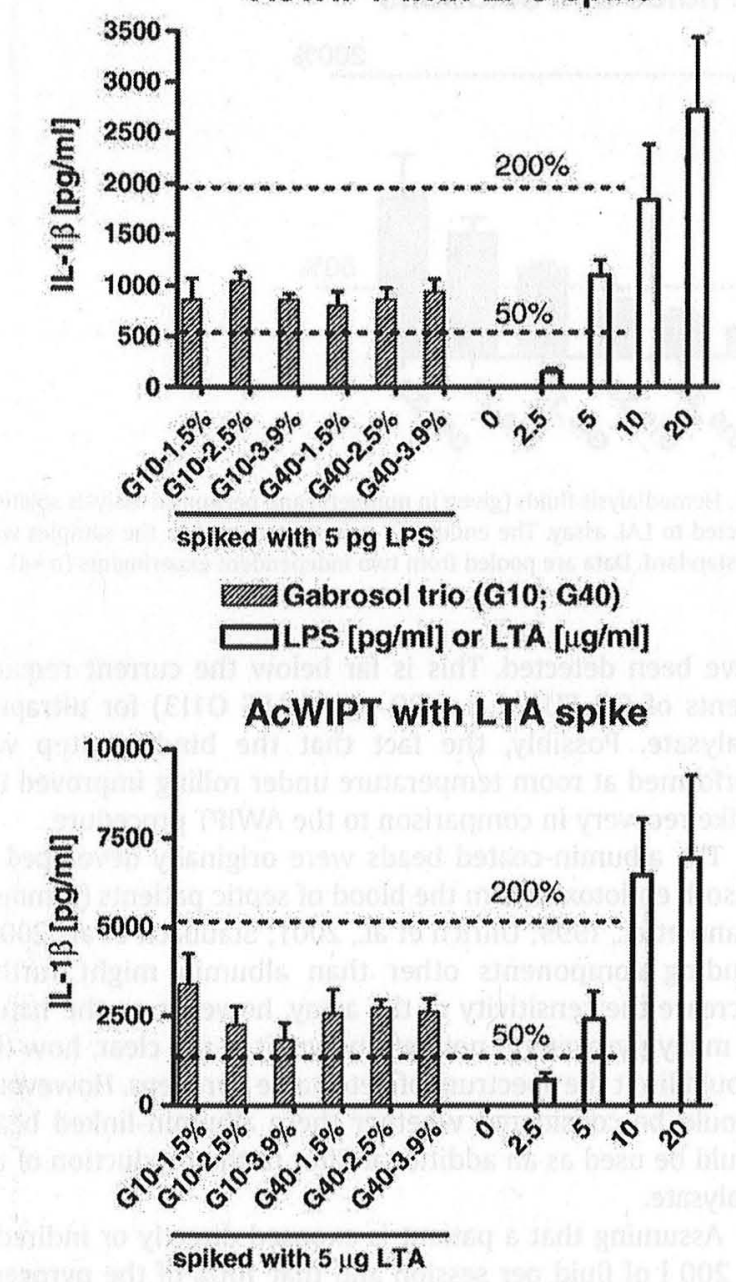

Fig. 4. Spike recovery in reconstituted peritoneal dialysis solution. $50 \mathrm{ml}$ of peritoneal dialysis solutions spiked with $5 \mathrm{pg}$ LPS (E. coli O-113), or $5 \mu \mathrm{g}$ LTA (S. aureus) were subjected to the AcWIPT procedure. "G10" and "G40" denote Gambro solutions 10 and 40 ; the suffixed percentage numbers give the concentrations of glucose. Dotted lines show 50\% and 200\% retrieval of induced IL-1 $\beta$ amounts compared to corresponding concentration response curves; data are triplicates representing three independent experiments. IL$1 \beta$ induction by all unspiked samples was below the detection limit of the ELISA (3.4 pg/ml).

of the concentrates. The working dilutions produced excellent spike recovery in all solutions, whereas the 1:3 dilution also affords a sufficient spike recovery (Fig. 3). Thus this assay is sensitive to $0.3 \mathrm{pg} / \mathrm{ml}$ LPS in diluted hemodialysis fluids, whereas no dilution is necessary for detection of LTA in these fluids.

Reconstituted peritoneal dialysis solutions were also subjected to the whole blood assay (Fig. 4). In the AcWIPT assay, the peritoneal dialysis solutions did not interfere either with LPS or with LTA spike recovery: the amounts of IL-1 $\beta$ induced upon stimulation with $0.1 \mathrm{pg} / \mathrm{ml}$ spike (AcWIPT) or $50 \mathrm{pg} / \mathrm{ml}$ spike (AWIPT) are not significantly different from those induced in saline.

Notably, neither the hemodialysis fluids nor peritoneal fluid solutions induced detectable IL-1 $\beta$ without being spiked with pyrogens.

\subsection{Detection of living microbes using IPT by AcWIPT}

In addition to the experiments aiming to recover pyrogens (LPS, LTA) we also tested the adsorption and detection of living microbes, i.e. E. coli and S. aureus. As shown in Fig. 5 there was a tenfold improvement of the detection limits for both specimens in the AcWIPT over the IPT assay.

\subsection{Recovery of low concentrated LPS spike with LAL}

Hemodialysis and peritoneal dialysis fluids and solutions were spiked in working concentrations with $1 \mathrm{pg} / \mathrm{ml} \mathrm{LPS}$ and the spike recovery was tested in a commercially available LAL kit. The spike could be recovered in six of twelve spiked samples. In the other six samples the recovery was below the $50 \%$ marker (Fig. 6 ).

\section{Discussion}

Some outbreaks of sterile peritonitis in dialysis patients have been traced back to endotoxin contaminations
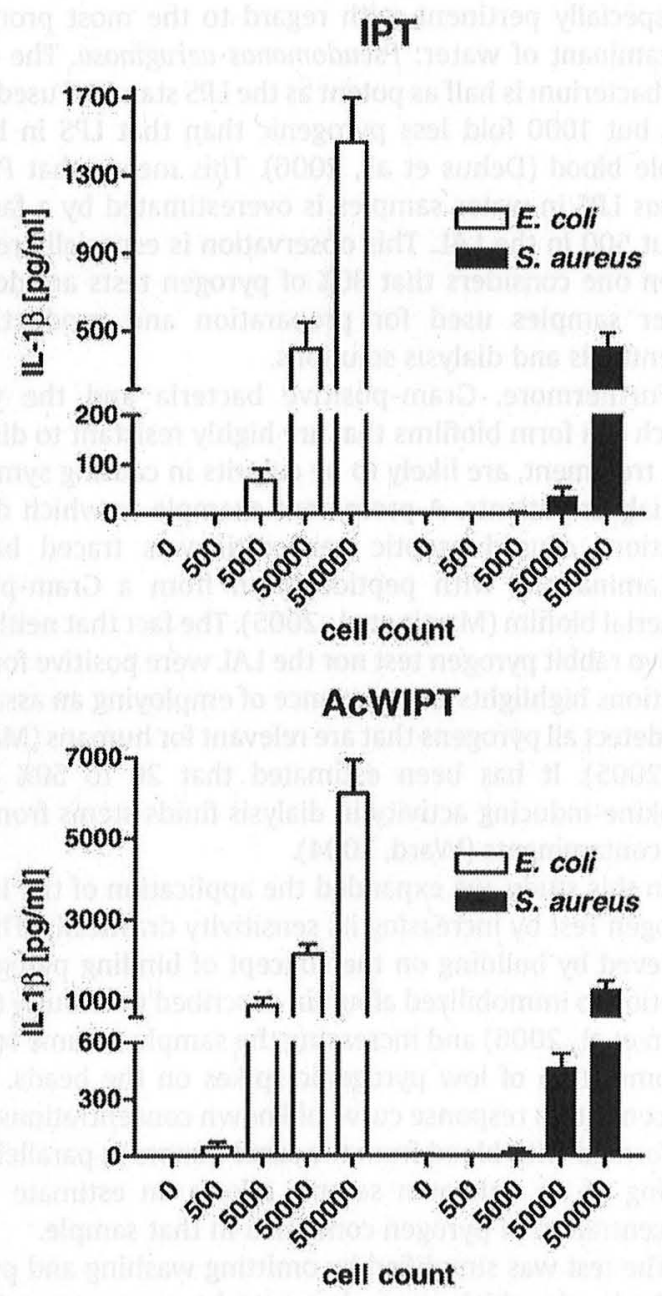

Fig. 5. Adsorption and recovery of live E. coli and S. aureus by IPT and AcWIPT. Live $E$. coli and $S$. aureus bacteria were subjected in different concentrations ( $n=3$, multiple IPT experiments, three independent AcWIPT assays) to the IPT and AcWIPT assays; detection of IL- $1 \beta$ indicates the immune recognition of the specimens. 


\section{LAL assay: spiked dialysis fluids and solutions}

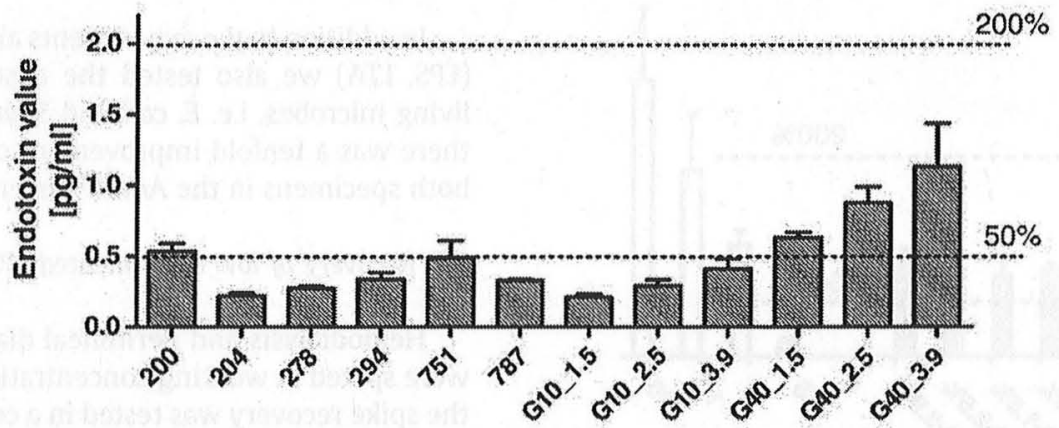

Fig. 6. Spike recovery in hemodialysis and peritoneal dialysis fluids and solutions by LAL. Hemodialysis fluids (given in numbers) and peritoneal dialysis solutions (G10's and G40's) were spiked in working concentration with $1 \mathrm{pg} / \mathrm{ml}$ LPS and subjected to LAL assay. The endotoxin values recovered in the samples were calculated by comparison with the concentration response curve of the LAL endotoxin standard. Data are pooled from two independent experiments $(n=4)$.

(Karanicolas et al., 1977; Mangram et al., 1998). Although variants of the LAL test have a sensitivity of about $3 \mathrm{pg} / \mathrm{ml}$ LPS, this test does not detect pyrogens other than LPS or reflect the different pyrogenic activity of LPS from different species. This is especially pertinent with regard to the most prominent contaminant of water: Pseudomonas aeruginosa. The LPS of this bacterium is half as potent as the LPS standard used in the LAL, but 1000 fold less pyrogenic than that LPS in human whole blood (Dehus et al., 2006). This means that Pseudomonas LPS in water samples is overestimated by a factor of about 500 in the LAL. This observation is especially relevant when one considers that $80 \%$ of pyrogen tests are done on water samples used for preparation and production of parenterals and dialysis solutions.

Furthermore, Gram-positive bacteria and the yeasts, which can form biofilms that are highly resistant to disinfection treatment, are likely to be culprits in causing symptoms in dialysis patients. A prominent example in which dialysis solutions caused aseptic peritonitis was traced back to contaminations with peptidoglycan from a Gram-positive bacterial biofilm (Martis et al., 2005). The fact that neither the in vivo rabbit pyrogen test nor the LAL were positive for these solutions highlights the relevance of employing an assay that can detect all pyrogens that are relevant for humans (Martis et al., 2005). It has been estimated that 20 to $50 \%$ of the cytokine-inducing activity in dialysis fluids stems from nonLPS contaminants (Ward, 2004).

In this study, we expanded the application of the In vitro Pyrogen Test by increasing its sensitivity drastically. This was achieved by building on the concept of binding pyrogens in solution to immobilized albumin described previously (Daneshian et al., 2006) and increasing the sample volume to allow accumulation of low pyrogenic spikes on the beads. A fine concentration response curve of known concentrations of LPS performed with blood from the same donors in parallel to the testing of an unknown sample allows an estimate of the concentration of pyrogen contained in that sample.

The test was simplified by omitting washing and priming of the beads which were shown to be unnecessary for the detection of LPS or of LTA from Gram-positive bacteria and the yeast extract zymosan. Spike recovery of only 5 pg LPS in $50 \mathrm{ml}$ of any of the dialysis solutions measured was possible, proving that a concentration of at least $0.1 \mathrm{pg} / \mathrm{ml}$ LPS would have been detected. This is far below the current requirements of $0.3 \mathrm{EU} / \mathrm{ml}$ (i.e. $30 \mathrm{pg} / \mathrm{ml}$ LPS 0113) for ultrapure dialysate. Possibly, the fact that the binding step was performed at room temperature under rolling improved the spike recovery in comparison to the AWIPT procedure.

The albumin-coated beads were originally developed to adsorb endotoxin from the blood of septic patients (Zimmermann et al., 1999; Ullrich et al., 2001; Staubach et al., 2003). Binding components other than albumin might further increase the sensitivity of the assay, however, as the nature of many pyrogens is not yet known, it is not clear, how this would limit the spectrum of detectable pyrogens. However, it should be considered whether these albumin-linked beads could be used as an additional filter in the production of the dialysate.

Assuming that a patient is exposed directly or indirectly to $200 \mathrm{I}$ of fluid per session and that $100 \%$ of the pyrogenic load passes the dialysis membrane, dialysis fluid that does not induce cytokine release in an AcWIPT assay with a sensitivity of $0.1 \mathrm{pg} / \mathrm{ml} \mathrm{LPS}$ (i.e. $0.001 \mathrm{EU} / \mathrm{ml}$ ), would contain a total pyrogenic burden of less than 2000 endotoxin equivalent units (EEU). Assuming a body weight of $70 \mathrm{~kg}$, this amounts to $3 \mathrm{EEU} / \mathrm{kg}$. Pyrogen tests for parenterals must be able to detect pyrogenic contaminations that would result in $5 \mathrm{EEU} / \mathrm{kg}$ as this is considered the fever threshold. This comparison demonstrates that only now, for the first time, the AcWIPT procedure allows pyrogen detection in dialysis fluids with a sensitivity that is high enough to truly rule out a systemic fever reaction. Regular testing of dialysis fluids with a method of this sensitivity combined with the use of a modern dialysis membrane that is less permeable to pyrogens could ensure the safety and preserve the health of dialysis patients.

\section{Acknowledgements}

We thank Armin Günther and Heidrun Leisner for their contributions to the work and Dr. Frank Hacket and Dr. Veit Otto from Fresenius Hemocare for supply of the albumincoupled beads and priming solution and for helpful discussions. A. Wendel and T. Hartung are holding patents for the IPT technology. A patent for the AWIPT/AcWIPT method is pending ( $T$. Hartung). The authors have no conflict of interest. 


\section{References}

Daneshian, M., Guenther, A., Wendel, A., Hartung, T., von Aulock, S., 2006. In vitro pyrogen test for toxic or immunomodulatory drugs. J. Immunol. Methods 313, 169.

Darveau, R.P., Pham, T.T., Lemley, K., Reife, R.A., Bainbridge, B.W., Coats, S.R. Howald, W.N., Way, S.S., Hajjar, A.M., 2004. Porphyromonas gingivalis lipopolysaccharide contains multiple lipid A species that functionally interact with both toll-like receptors 2 and 4 . Infect. Immun. 72, 5041.

Dehus, O., Hartung, T., Hermann, C., 2006. Endotoxin evaluation of eleven lipopolysaccharides by whole blood assay does not always correlate with Limulus amebocyte lysate assay. J. Endotoxin Res. 12, 171.

Dinarello, C.A., 2004. Infection, fever, and exogenous and endogenous pyrogens: some concepts have changed. J. Endotoxin Res. 10, 201.

Fennrich, S., Fischer, M., Hartung, T., Lexa, P., Montag-Lessing, T., Sonntag, H.G., Weigandt, M., Wendel, A., 1999. Detection of endotoxins and other pyrogens using human whole blood. Dev. Biol. Stand. 101, 131.

Hartung, T., Wendel, A., 1996. Detection of pyrogens using human whole blood. In Vitro Toxicol. 9, 353.

Hoffmann, S., Peterbauer, A., Schindler, S., Fennrich, S., Poole, S., Mistry, Y. Montag-Lessing, M., et al., 2005. International validation of nove pyrogen tests based on human monocytoid cells. J. Immunol. Methods $298,191$.

Karanicolas, S., Oreopoulos, D.G., Izatt, S., Shimizu, A., Manning, R.F., Sepp, H., de Veber, G.A., Darby, T., 1977. Epidemic of aseptic peritonitis caused by endotoxin during chronic peritoneal dialysis. N. Engl. J. Med. 296, 1336.

Kindinger, I., Daneshian, M., Baur, H., Gabrio, T., Hofmann, A., Fennrich, S., von Aulock, S., Hartung, T., 2005. A new method to measure air-borne pyrogens based on human whole blood cytokine response. J. Immunol. Methods 298, 143.

Ledebo, 1., 2004. Ultrapure dialysis fluid-direct and indirect benefits in dialysis therapy. Blood Purif. 22 (Suppl. 2), 20.

Mangram, A.J., Archibald, L.K., Hupert, M., Tokars, J.I., Silver, L.C., Brennan, P. Arduino, M., Peterson, S., Parks, S., Raymond, A., McCullough, M., Jones, M., Wasserstein, A., Kobrin, S., Jarvis, W.R., 1998. Outbreak of sterile peritonitis among continuous cycling peritoneal dialysis patients. Kidney Int. 54, 1367.

Martis, L., Patel, M., Giertych, J., Mongoven, J., Taminne, M., Perrier, M.A., Mendoza, O., Goud, N., Costigan, A., Denjoy, N., Verger, C., Owen Jr., W.F., 2005. Aseptic peritonitis due to peptidoglycan contamination of pharmacopoeia standard dialysis solution. Lancet 365, 588.

Mazzotti, F., Beuttler, J., Zeller, R., Fink, U., Schindler, S., Wendel, A., Hartung, T., von Aulock, S., 2007. In vitro pyrogen test-a new test method for solid medical devices. J. Biomed. Mater. Res. A 80, 276.

Morath, S., Geyer, A., Hartung, T., 2001. Structure-function relationship of cytokine induction by lipoteichoic acid from Staphylococcus aureus. J. Exp. Med. 193, 393.

Poole, S., Mussett, M.V., 1989. The International Standard for Endotoxin: evaluation in an international collaborative study. J. Biol. Stand. 17, 161

Schindler, S., Spreitzer, I., Loschner, B., Hoffmann, S., Hennes, K., Halder, M., Brugger, P., Frey, E., Hartung, T., Montag, T., 2006. International validation of pyrogen tests based on cryopreserved human primary blood cells. J. Immunol. Methods 316, 42.

Staubach, K.H., Boehme, M., Zimmermann, M., Otto, V., 2003. A new endotoxin adsorption device in Gram-negative sepsis: use of immobilized albumin with the MATISSE adsorber. Transfus. Apheresis Sci. 29, 93.

Ullrich, H., Jakob, W., Frohlich, D., Rothe, G., Prasser, C., Drobnik, W., Taeger, K., Meier-Hellmann, A., Reinhart, K., Zimmermann, M., Schmitz, G., 2001. A new endotoxin adsorber: first clinical application. Ther. Apher. 5, 326.

Walev, J. Klein, J. Husmann, M. Valeva, A, Strauch, S, Wirtz, H., Weichel, 0. Bhakdi, S., 2000. Potassium regulates IL-1 beta processing via calciumindependent phospholipase A2. J. Immunol. 164, 5120.

Ward, R.A., 2004. Ultrapure dialysate. Semin. Dial. 17, 489.

Zimmermann, M. (2000) In vitro Untersuchungen zum Adsorptionsverhalten eines Endotoxinadsorbers auf der Basis von immobilisiertem Humanalbumin. In. Ph. D. thesis, University of Saarbrücken.

Zimmermann, M., Busch, K., Kuhn, S., Zeppezauer, M., 1999. Endotoxin adsorbent based on immobilized human serum albumin. Clin. Chem. Lab. Med. 37, 373. 
